\title{
Inverse Method and Algorithm to Retrieve the Heat Diffusivity of Solid Samples in a Non-Destructive Way
}

\author{
Ziemowit Ostrowski, Arkadiusz Ryfa, Zbigniew Buliński, Wojciech P. Adamczyk \\ Institute of Thermal Technology, Silesian University of Technology \\ Konarskiego 22, 44-100 Gliwice, Poland \\ ziemowit.ostrowski@polsl.pl; arkadiusz.ryfa@polsl.pl; zbigniew.bulinski@polsl.pl; wojciech.adamczyk@polsl.pl
}

\section{Extended Abstract}

Classical, well-established method of heat diffusivity measurement, e.g. Parker flash method [1], is expensive and time-consuming. The experimental facilities used to measure the properties are designed to handle specially manufactured samples of an investigated material. This simplifies the solution of the resulting inverse problem but makes the approach material destructive.

Presented approach deals with the non-destructive technique of evaluation of thermal diffusivity that resorts to the principle of active thermography. The idea relies on short heating of a small surface area of the investigated body by a laser impulse and recording the changes of the temperature field by an infrared (IR) camera. Based on the measured surface temperature history, an inverse problem of heat transfer (IHTP) is solved to retrieve the values of the heat diffusivity.

In the current approach, as a direct solver of IHTP, the usage of Computational Fluid Dynamics (CFD) model of the material sample is proposed. The major drawback of such approach is long computation time which considerable limits it's practical application. As the solution of IHTP requires numerous runs of the direct solver, a reduced order model (ROM) was proposed instead of full CFD direct solution. The Proper Orthogonal Decomposition (POD) empirical vectors were used as approximation basis of ROM, while the dependency of the ROM on input parameters is obtained by means of Radial Basis Functions (RBF). The POD-RBF trained model is proven to be a fast yet accurate approach to build ROM $[2,3]$.

In the current research, a novel method of selection of time- and spatial- distribution of measurement points is proposed. This improves previously used approaches [4] and make the method more robust and universal.

The developed method can retrieve heat conductivity with high accuracy. The results obtained using proposed approach were validated using additional experimental data obtained in commercially available apparatus Netzsch LFA 457 (NETZSCH-Gerätebau GmbH, Germany). Good accuracy has been achieved.

\section{Acknowledgments}

The research has been supported by National Science Centre (Poland) within SONATA scheme under contract No 2014/15/D/ST8/02620.

\section{References}

[1] W. J. Parker, R. J. Jenkins, C. P. Butler, G. L. Abbott, "A Flash Method of Determining Thermal Diffusivity, Heat Capacity, and Thermal Conductivity," J Appl Phys, vol.32, no. 9, pp. 1679-1684, 1961.

[2] Z. Ostrowski, R. A. Bialecki, A. J. Kassab, "Solving inverse heat conduction problems using trained POD-RBF network inverse method," Inv Probl Sci Eng, vol. 16, no. 1, pp. 39-54, 2008.

[3] C. A. Rogers, A. J. Kassab, E. A. Divo, Z. Ostrowski, R. A. Bialecki, "An inverse POD-RBF network approach to parameter estimation in mechanics," Inv Probl Sci Eng, vol. 20, no. 5, pp. 749-767, 2012.

[4] W. Adamczyk, Z. Ostrowski, "Retrieving thermal conductivity of the solid sample using reduce order model inverse approach," Int J Num Meth Heat Fluid Flow, vol. 27, no. 3, pp. 729-739, 2017. 